\title{
Geographic clustering of elderly people with above-norm anthropometric measurements and blood chemistry
}

\author{
Carlos Mena, ${ }^{1}$ Eduardo Fuentes, ${ }^{2,3}$ Yony Ormazábal, ${ }^{1}$ Iván Palomo ${ }^{2}$ \\ ${ }^{1}$ Geomatics Center, University of Talca; ${ }^{2}$ Platelet Research Laboratory, Department of Clinical Biochemistry \\ and Immunohaematology, Faculty of Health Sciences, University of Talca; \\ ${ }^{3}$ Scientific Multidisciplinary Nucleus, University of Talca, Chile
}

\begin{abstract}
The global percentage of people over 60 is strongly increasing and estimated to exceed $20 \%$ by 20,150 , which means that there will be an increase in many pathological conditions related to aging. Mapping of the location of aging people and identification of their needs can be extremely valuable from a social-economic point of view. Participants in this study were 148 randomly selected adults from Talca City, Chile aged 60-74 at baseline. Geographic information systems (GIS) analyses were performed using ArcGIS software through its module Spatial Autocorrelation. In this study, we demonstrated that elderly people show geographic clustering according to above-norm results of anthropometric measurements and blood chemistry. The spatial identifications found would facilitate exploring the impact of treatment programmes in communities where many aging people live, thereby improving their quality of life as well as reducing overall costs.
\end{abstract}

Correspondence: Carlos Mena, Geomatics Center, University of Talca, 2 Norte, 685 Talca, Chile.

Tel: +56.71 .2200200 .

E-mail: cmena@utalca.cl

Key words: Geographic information system; Moran's I; Getis-Ord Gi*; Aging; Cardiovascular parameters.

Conflict of interest: the authors declare no potential conflict of interest.

Acknowledgements: this work was funded by Interdisciplinary Excellence Research Program on Healthy Aging (PIEI-ES).

Received for publication: 13 October 2016.

Revision received: 5 February 2017.

Accepted for publication: 6 February 2017.

CCopyright C. Mena et al., 2017

Licensee PAGEPress, Italy

Geospatial Health 2017; 12:523

doi:10.4081/gh.2017.523

This article is distributed under the terms of the Creative Commons Attribution Noncommercial License (CC BY-NC 4.0) which permits any noncommercial use, distribution, and reproduction in any medium, provided the original author(s) and source are credited.

\section{Introduction}

The global percentage of people over 60 is currently $11 \%$ with an estimated climb to $21 \%$ by 2050 (Bloom et al., 2011). This means an increase in many pathological conditions related to aging (Vermeulen et al., 1998; Fjell and Walhovd, 2010; Bueno et al., 2014) and may partly explain the higher incidence of cardiovascular disease (CVD) in older adults (Kovacic et al., 2011; Raskob et al., 2014; Sarink et al., 2014). While aging is considered an independent risk factor for the development of CVD, older adults are also at high risk for developing other chronic diseases (Lopes et al., 2012). Mena et al. $(2015,2016)$ showed the importance of the role of environmental factors such as parks and markets in the development of cardiovascular risk. Mapping of the location of aging people and identification of their needs can therefore be of high value from the social-economic point of view.

Geographic Information Systems (GIS) are part of the more general and modern concept denominated geo-information, or geomatics, that integrates GIS with disciplines, such as global positioning system (GPS), remote sensing (RS), cartography, geodesy and similar approaches (Gonzalez, 2013). The application of GIS technology includes fields like forestry, agriculture, mining, urban planning, network management, geo-marketing, ecology, transportation, geology, land planning, public health, etc. (Tanser, 2002). In the field of public health, GIS have multiple potential applications in areas such a disease surveillance, risk analysis, health access and planning, community health profiling, which takes into account various settings like countryside, city, built environment and other neighbourhoods (Shaw, 2012). In addition, the GIS potential has been identified by experts in relation to improved understanding of complex health issues and to support the design and evaluation of effective programmes and strategies based on population data (Carroll et al., 2014). The purpose of this study was to examine a cohort of elderly people, controlling their body measurements and general blood chemistry values, and collate this information to find out if there is any degree of geographic clustering that could be useful from the point of view of health provision.

\section{Materials and Methods}

\section{Study site and participants}

The study took place in the city of Talca, which is located about $250 \mathrm{~km}$ south of Santiago and is the capital of both Talca Province and Maule Region. Considering a significance level of 
$5 \%$ and a power of $80 \%$, a representative sample of 148 participants aged 60-74 years at baseline (98 women and 50 men) were randomly selected from elderly people in Talca City, Chile. The sample selected represented close to $1 \%$ of Talca's population. Only persons free of clinical cardiovascular disease at baseline were enrolled. The variables included postal address, weight, height, waist circumference, body mass index (BMI), blood pressure and blood chemistry with special reference to total lipoproteins, total cholesterol, low-density lipoprotein cholesterol (LDLC), high-density lipoprotein cholesterol (HDL-C), triglycerides, glycaemia and uric acid. Additional details on the design of study are provided elsewhere (Palomo et al., 2007).

\section{Geographic information system application}

A geo-referenced database was built in ArcGIS software version 10 (ESRI, Redlands, CA, USA). This database provided all relevant geo-information stored as digital layers representing roads, urban blocks, green areas (squares, avenues, parks, etc.). We also used SPOTMaps satellite-generated images (http://www.intelligence-airbusds.com/spotmaps/). All this information was organised as independent information layers and conformed a base map where every single participant was geo-located according to the home address given during the medical evaluation. GIS coverage was created using georeferenced points as participant-representative elements. This coverage was the main input data for the spatial autocorrelation analysis (Mena et al., 2015, 2016). Moran's I was calculated by means of ArcGIS's module Analyzing Patterns.

\section{Spatial correlation statistics}

\section{Moran's I}

Global statistics can help identify spatial association in a large group of values registered for the spatial location of individuals (Fotheringham and Brunsdon, 1999). We used Moran's I to analyse the correlation among observations of neighbouring participants in order to detect whether the spatial distribution was clustered, dispersed or random. Moran's $I$ was calculated according to Chen et al. (2015):

$$
I=\frac{n * \sum_{i=1}^{n} \sum_{j=1}^{n} w_{i j}\left(x_{i}-x\right)\left(x_{j}-x\right)}{\left(\sum_{i=1}^{n} \sum_{j=1}^{n} w_{i j}\right) * \sum_{i=1}^{n}\left(x_{i}-x\right)^{2}}, i \neq j
$$

where $n$ is the number of participants, $x$ the average value for some medical measurement, $x_{i}$ and $x_{j}$ the medical measurement for participant $i$ and $j$, respectively, and $w_{i j}$ the spatial weight between participants $i$ and $j$ (Ferguson and Korfmacher, 1997). The analyses were performed using ArcGIS software through its module Analyzing Patterns according to Ferguson and Korfmacher (1997). The tool calculates the value of Moran's $I$ and also a Z-score and P value, which are both useful to evaluate the significance of the index. Z test (z-score) is usually used as a hypothesis test to determine whether a spatial clustering exists and its calculation is made by the formula:
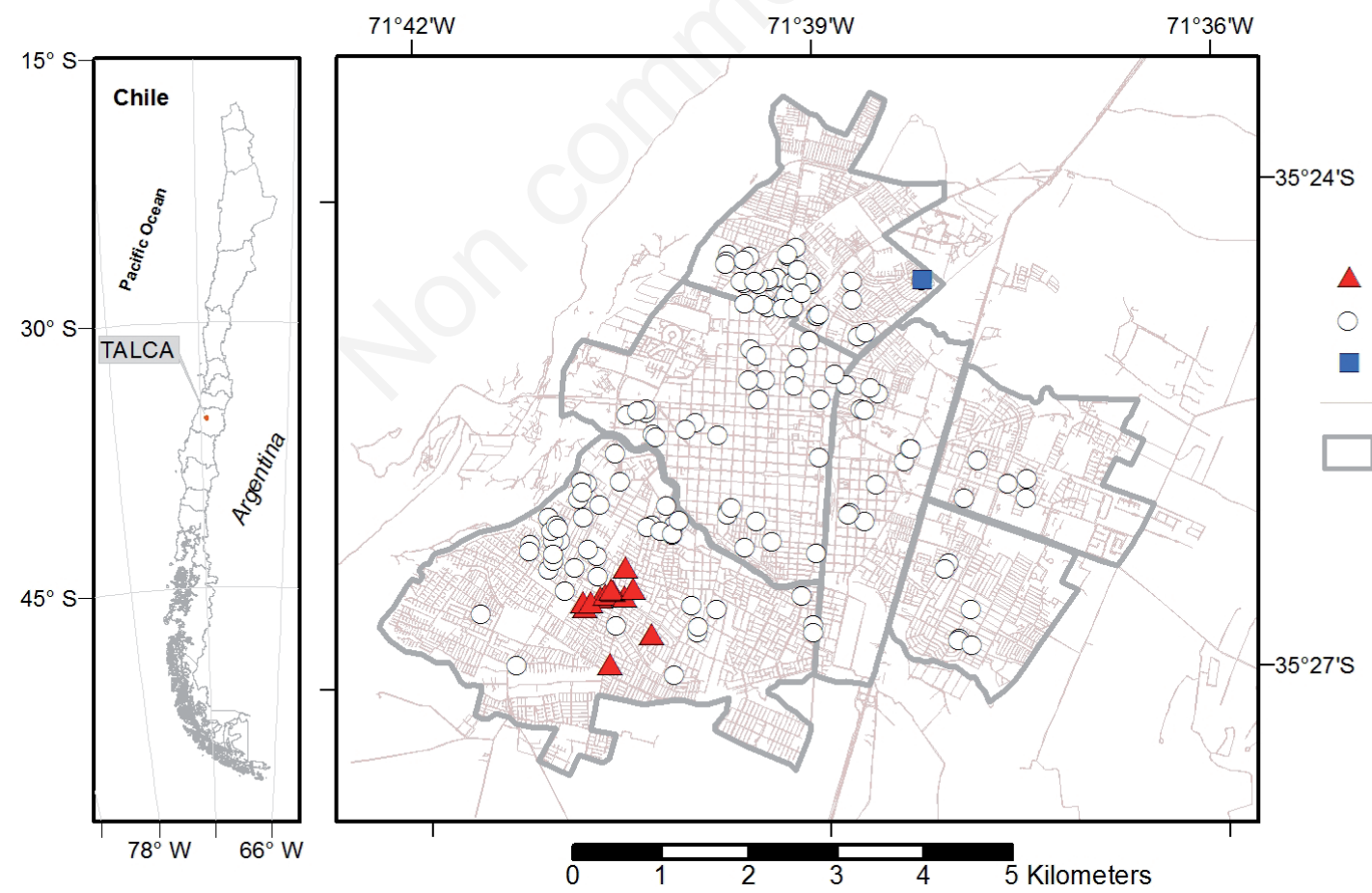

\section{LEGEND}

- Hot Spot (High Values)

Not Clustered

Cold Spot (Low Values)

Streets

Urban Boundary

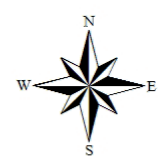

Elipsoide: WGS 84

Datum: WGS 84

Figure 1. Getis-Ord Gi* statistic result for weight values. The figure clearly shows a hotspot for the weight variable located in the southwestern part of the city centre, an area associated with medium-low income level, where the mean registered weight is $76.65 \mathrm{~kg}$. A small coldspot is also seen to the northeast (the old city), where the mean weight value is $56.75 \mathrm{~kg}$. 


$$
z_{I}=\frac{I-E[I]}{\sqrt{V[I]}}
$$

$E[I]=-1 /(\mathrm{n}-1)$ and $V[I]=\mathrm{E}\left[\mathrm{I}^{2}\right]-\mathrm{E}[\mathrm{I}]^{2}$. The null hypothesis confirms that measure values are randomly distributed in the study area. For a $95 \%$ of confidence level (CL), the critical z-score values should be between -1.96 and +1.96 standard deviations (SD) with an associated $\mathrm{P}$ value of 0.05 . Thus, a value outside this range rejects the null hypothesis and proves that a significant spatial autocorrelation among the measurement values for the study area exists. We used a threshold distance of $700 \mathrm{~m}$ search radio for the analyses. Finally, Moran's $I$ range between -1 to +1 where a value near 0 indicates lack of spatial clustering, so values registered for medical measurement of patients at one location do not depend on neighbourhood or local conditions; meanwhile, values near +1 indicate that measurement values tend to cluster, while values near -1 imply that higher and lower measurement values are interspersed in the analysed area (Zhao et al., 2013).

\section{Local indicators of spatial association statistics}

Local indicators of spatial association (LISA) statistics can help to identify the local spatial association between a value registered for a participant spatially located and its neighbours, given a specified distance from the observation (Roger et al., 2012). We used the $\mathrm{G}_{\mathrm{i}}{ }^{*}$ local statistic (Ord and Getis, 1995) to analyse the spatial correlation among observations of neighbouring participants in order to identify statistically significant spatial clusters of high values (hotspots) and low values (coldspots). Getis-Ord $\mathrm{G}_{\mathrm{i}}{ }^{*}$ statistic was calculated by formula:

$$
\begin{aligned}
& G_{i}^{*}=\frac{\sum_{j=1}^{n} w_{i, j} x_{j}-\bar{X} \sum_{j=1}^{n} w_{i, j}}{S \sqrt{\frac{\left.\mid n \sum_{j=1}^{n} w_{i, j}^{2}-\left(\sum_{j=1}^{n} w_{i, j}\right)^{2}\right]}{n-1}}} \mathrm{G}_{\mathrm{i}}^{*}= \\
& \sum_{\mathrm{j}=1}^{\mathrm{n}} \mathrm{w}_{\mathrm{i}, \mathrm{j}} \mathrm{x}_{\mathrm{j}}-\overline{\mathrm{X}} \sum_{\mathrm{j}=1}^{\mathrm{n}} \mathrm{w}_{\mathrm{i}, \mathrm{j}} \\
& S \sqrt{\frac{\left[\mathrm{n} \sum_{j=1}^{\mathrm{n}} \mathrm{w}_{\mathrm{i}, \mathrm{j}}{ }^{2}-\left(\sum_{\mathrm{j}=1}^{\mathrm{n}} \mathrm{w}_{\mathrm{i}, \mathrm{j}}\right)^{2}\right]}{\mathrm{n}-1}}
\end{aligned}
$$

where $x_{j}$ is the attribute value for participant $j, w_{i, j}$ the spatial weight between participant $i$ and $j$, while $n$ corresponds to the total number of participants. $\overline{\mathrm{X}}$ and $S$ were calculated by equations 4 and 5 below:

$$
\bar{X}=\frac{\sum_{j=1}^{n} x_{j}}{n}
$$

$$
S=\sqrt{\frac{\sum_{j=1}^{n} x_{j}^{2}}{n}-(\bar{X})^{2}}
$$

The analyses were applied using ArcGIS software through its module Mapping Cluster to those medical measurements that show clustered condition by Moran's $I$. The $\mathrm{G}_{i}{ }^{*}$ statistic is a Z-score so no further calculations were needed. Then $z$-score and p-value were used to evaluate where participants with either high or low values clustered spatially. A statistically significant hotspot or coldspot is identified when a participant has a high (or low) value and is surrounded by other participants with similar (high or low) values as well. A participant with a positive $\mathrm{z}$-score corresponds to a spatial clustering of high values and participant with negative z-

Table 1. Characteristics of the study population $(n=148)$.

\begin{tabular}{lccc} 
Variable & Mean & SD & Norm \\
Age* (years) & 66 & 4 & \\
Height $(\mathrm{cm})$ & 160 & 20 & \\
\hline Weight $(\mathrm{kg})$ & 72.6 & 13.6 & \\
BMI $\left(\mathrm{kg} / \mathrm{m}^{2}\right)$ & 29.1 & 5.5 & $\leq 25^{\circ}$ \\
\hline Waist circumference $(\mathrm{cm})$ & 94.6 & 11.8 & \\
Blood pressure $(\mathrm{mm} \mathrm{Hg})$ & 144.6 & 22.4 & $<120$ \\
\hline Uric acid $(\mathrm{mg} / \mathrm{mL})$ & 5.2 & 1.6 & $3.5-7.0$ \\
Glycaemia $(\mathrm{mg} / \mathrm{dL})$ & 105.6 & 27.1 & $<100$ \\
\hline Total cholesterol (mg/dL) & 208.9 & 38.4 & $>200$ \\
LDL-C (mg/dL) & 121.7 & 39.7 & $<100$ \\
\hline HDL-C (mg/dL) & 52.6 & 14.8 & $>60$ \\
Triglycerides (mg/dL) & 161.1 & 87.1 & $\leq 150$ \\
\hline
\end{tabular}

SD, standard deviation; BMI, body mass index; LDL-C, low-density lipoprotein cholesterol; HDL-C, highdensity lipoprotein cholesterol. *Age range of the study subjects=60-70 years; ' ${ }^{\circ}$ between $>25$ and $<30=$ overweight, $\geq 30=$ obesity; "systolic pressure.

Table 2. Results of statistical analysis $(\mathbf{n}=148)$.

\begin{tabular}{lccc} 
Variable & Moran's $I$ & P & Z-score \\
Age* (years) & 0.082 & 0.145 & 1.456 \\
Height $(\mathrm{cm})$ & 0.026 & 0.479 & 0.724 \\
\hline Weight $(\mathrm{kg})$ & 0.118 & 0.040 & 2.051 \\
BMI $\left(\mathrm{kg} / \mathrm{m}^{2}\right)$ & 0.197 & 0.001 & 3.396 \\
\hline Waist circumference $(\mathrm{cm})$ & 0.163 & 0.005 & 2.800 \\
Blood pressure $^{\circ}(\mathrm{mm} \mathrm{Hg})$ & 0.043 & 0.405 & 0.832 \\
\hline Uric acid $(\mathrm{mg} / \mathrm{mL})$ & -0.036 & 0.620 & -0.495 \\
Glycaemia $(\mathrm{mg} / \mathrm{dL})$ & -0.005 & 0.974 & 0.031 \\
\hline Total cholesterol $(\mathrm{mg} / \mathrm{dL})$ & -0.035 & 0.638 & -0.470 \\
LDL-C (mg/dL) & -0.092 & 0.159 & -1.405 \\
\hline HDL-C $(\mathrm{mg} / \mathrm{dL})$ & -0.018 & 0.849 & -0.190 \\
Triglycerides $(\mathrm{mg} / \mathrm{dL})$ & 0.038 & 0.451 & 0.754
\end{tabular}

BMI, body mass index; LDL-C, low-density lipoprotein cholesterol; HDL-C, high-density lipoprotein cholesterol. *Age range of the study subjects $=60-70$ years; ${ }^{\circ}$ systolic pressure.

Table 3. Spatial autocorrelation (cluster) values.

\begin{tabular}{lcc} 
Variable & Low cluster & High cluster \\
Weight $(\mathrm{kg})$ & $53.4 \pm 4.1$ & $101.9 \pm 13.4$ \\
BMI $\left(\mathrm{kg} / \mathrm{m}^{2}\right)$ & $16.7 \pm 9.4$ & $38.37 \pm 3.2$ \\
\hline
\end{tabular}

BMI, body mass index. Values are expressed as mean \pm standard deviation. 

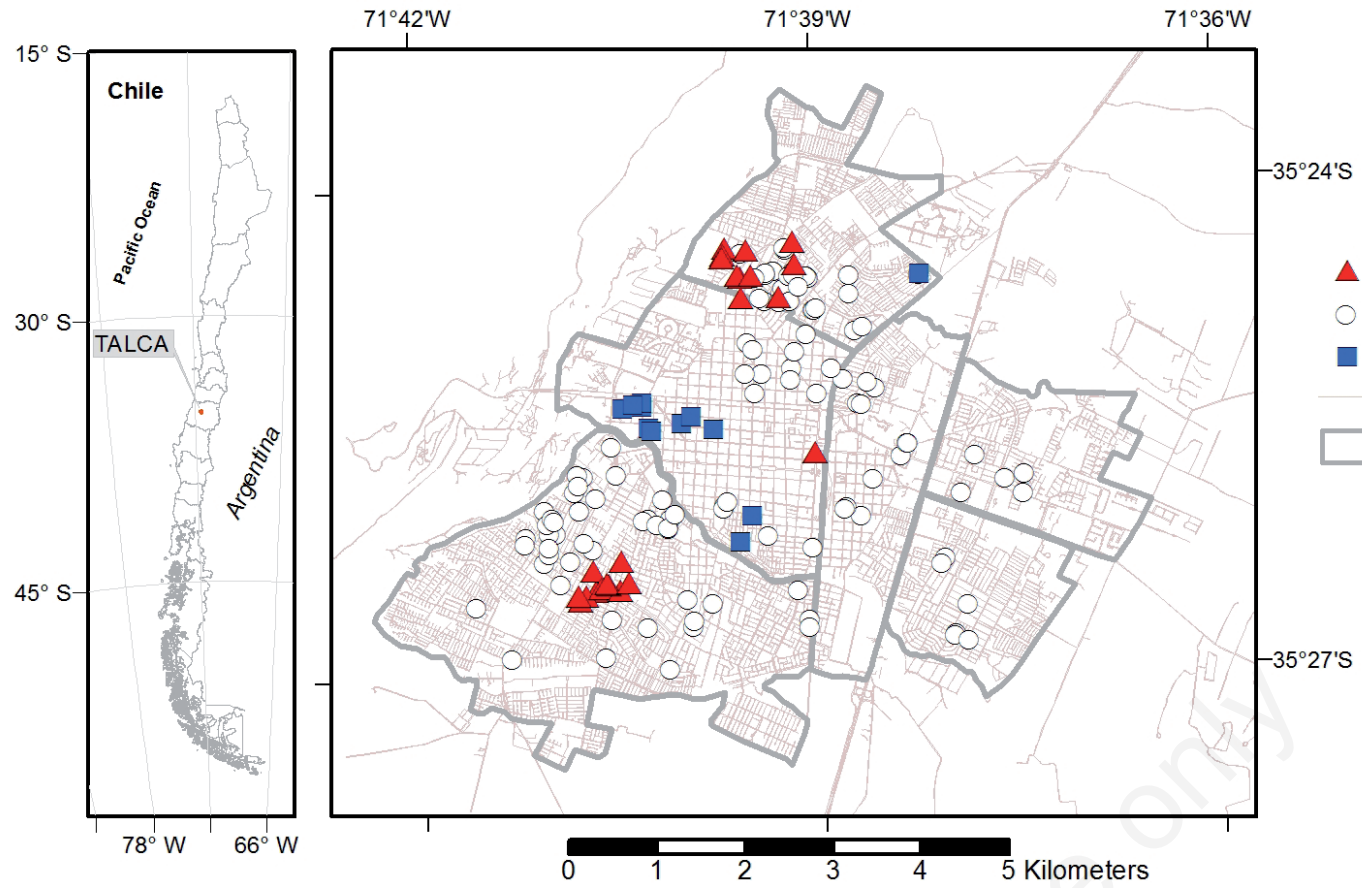

\section{LEGEND}

Hot Spot (High Values)

Not Clustered

Cold Spot (Low Values) Streets

Urban Boundary

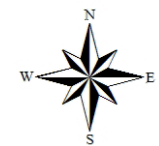

Elipsoide: WGS 84 Datum: WGS 84

Figure 2. Getis-Ord Gi* statistic result for waist values. Two hotspots referring to the waist circumference variable are located in the southwestern part of the city centre (same area as that with weight hotspot) but also to the northwest (both areas are characterised by medium-low income levels). Mean value for these places is $99.34 \mathrm{~cm}$. A small coldspot is also seen in the northeast (the old city), where the mean value is $88.25 \mathrm{~cm}$.
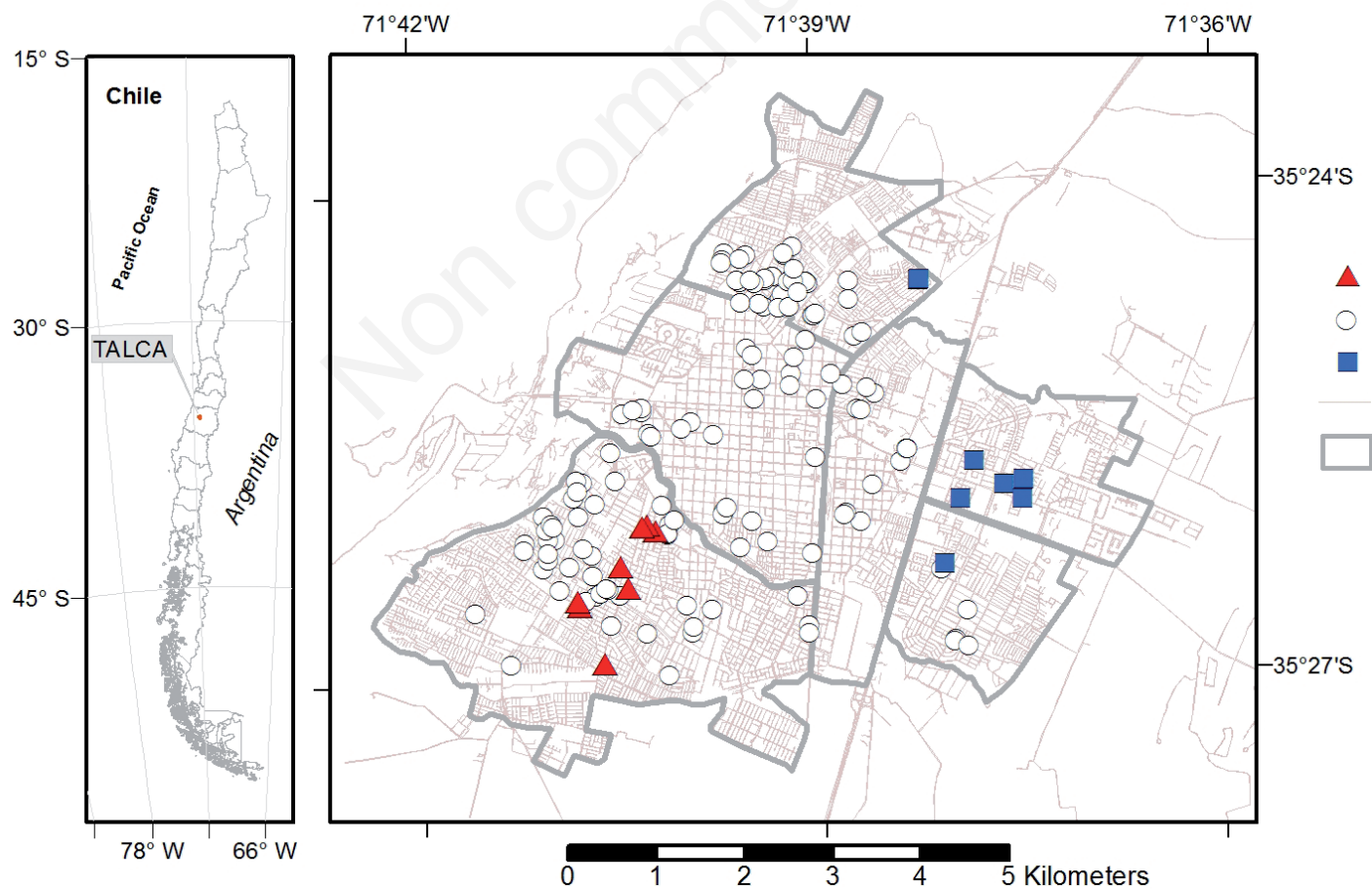

\section{LEGEND}

- Hot Spot (High Values) Not Clustered Cold Spot (Low Values) Streets Urban Boundary

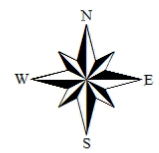

Elipsoide: WGS 84 Datum: WGS 84

Figure 3. Getis-Ord Gi* statistic result for body mass index (BMI) values. A BMI hotspot is located southwest of the city centre in the same area of hotspots registered for the weight and waist circumference variables. The BMI mean value for this area is $254.65 \mathrm{~kg} / \mathrm{m}^{2}$. Coldspots are located in the north and eastern part of the city, spatially coincident with an area of high-income where the mean value of BMI is $178.68 \mathrm{~kg} / \mathrm{m}^{2}$. 
score corresponds to a spatial clustering of low values. The higher (or lower) the $\mathrm{z}$-score, the more intense the clustering, while $\mathrm{z}$ scores near zero show no apparent spatial clustering (Getis and Aldstadt, 2004).

\section{Statistical analysis}

Mean \pm SDs were determined using SPSS version 17.0 (SPSS inc., Chicago, IL, USA). The GIS analyses were performed using ArcGIS software through its module Spatial Autocorrelation. $\mathrm{P}<0.05$ were considered significant.

\section{Results}

\section{Characterisation of the study population}

Table 1 shows the individual characteristics together with normal values seen in the population at large. As can be seen, the majority of the study subjects, showed above normal results of body measurements and blood chemistry as follows: BMI (79\%), blood pressure $(86 \%)$, glycaemia $(50 \%)$, total cholesterol $(62 \%)$ and triglycerides $(45 \%)$.

\section{Geographic information system and spatial autocorre- lation analysis}

Moran's I was calculated based on the geo-referenced ArcGIS database. The results of this statistical analysis are shown on Table 2. A significant positive spatial autocorrelation (cluster: $\mathrm{P}<0.05$ ) was reported for weight, waist circumference and BMI, the latter being the most clustered variable. The spatial autocorrelation (clustering tendency) according to weight and BMI stood out. As shown in Table 3, people were broadly grouped around low and high values with each of the two variables clustering at low and high levels. Getis-Ord Gi* statistic was calculated in order to identify statistically significant spatial clusters of high values (hotspots) and low values (coldspots) among neighbouring participants. The calculated variables were weight, waist circumference and BMI. The results of this local statistic are shown in Figures 13 .

\section{Discussion}

In this study, we have demonstrated for the first time that elderly people have a tendency to cluster according to some anthropometric measurements and blood chemistry levels that are above the norm. Thus, clustering as shown by GIS could help improving the quality of life by highlighting the need for exploration of the impact of treatment programmes in communities where aging people congregate. GIS is a relatively new concept in the geo-oriented sciences arising from the integration of cartography and information technologies (Bergquist and Tanner, 2012). The relational database model offered allows the linking of disparate types of geo-data by means of GIS software facilitating data management in this area of research (Wieczorek and Delmerico, 2009).

The future challenges of health-GIS integration mainly concern understanding and treatment of health problems that arise in different geographical areas (Fradelos et al., 2014). In this article, the integration of health-related data across different georeferenced areas in a city brought out novel insights how the health care system could be more involved by considering the residential distribution of the mainly aging population stratum with above-norm anthropometric measurements and blood chemistry. However, it is necessary to resolve methodological issues related to the number of cases of various ailments occurring in a geographic area as the numbers can be small and the people with health needs can be unevenly distributed. However, a better picture of this can be accomplished with efficient training in GIS concepts, practices and application besides a development focus on geomatics and health care integration.

In this study, we observed that when there was an increase or decrease in such body measurements as weight, BMI and waist circumference, elderly people were spatially autocorrelated (clustered) within limited areas, such as areas of 700-m radius. Spatial autocorrelation calculation is considered a useful technique for cluster mapping of regional health care problems, since it helps to analyse the geospatial correlation between aspects concerned with health care events (Tsai et al., 2009; Varga et al., 2013). The theory of spatial autocorrelation is an important part of geographical analysis and among the various approaches, Moran's $I$ is one of the most commonly used statistics due to its ability to explicitly identify spatial outliers (Sugumaran et al., 2009; Chen et al., 2015). Moran's I is a generalisation of Pearson's correlation coefficient, where an assessment of the correlation of a variable and its spatial location (cross-product between location similarity and attribute similarity) is performed (Zhao et al., 2013). The integration of spatial autocorrelation analyses, GIS and existing health data has provided public health officials with an efficient informatics tool that can be used to detect and study the prevalence of statistically significant hotspots with respect to many different medical issues (Toan et al., 2013), such as infectious disease (Bhunia et al., 2013), cancer incidence (Bhunia et al., 2013), intellectual disability (Goli et al., 2014), cardiac disease (Loughnan et al., 2008) and BMI (Duncan et al., 2012). In addition, results of GetisOrd Gi* statistic show spatial clustering of medical measurement registered for elderly people, specifically weight, waist circumference and BMI, where hotspots and coldspots were shown to be related to areas characterised by different income levels.

\section{Conclusions}

The results presented here support the potential for developing a GIS-related health care approach in tasks where the impact of cluster evaluation of human health issues can be enabled when map analysis is included. In this context, it is very important to create geo-databases associated with health care providers at the local, even micro level.

\section{References}

Bergquist R, Tanner M, 2012. Visual approaches for strengthening research, science communication and public health impact. Geospat Health 6:155-6.

Bhunia GS, Kesari S, Chatterjee N, Kumar V, Das P, 2013. Spatial and temporal variation and hotspot detection of kala-azar disease in Vaishali district (Bihar), India. BMC Infect Dis 13:1-12.

Bloom DE, Boersch-Supan A, McGee P, Seike A, 2011. Population aging: facts, challenges, and responses. Benefits Compensat Int $41: 1-22$ 
Bueno V, Sant'Anna O, Lord J, 2014. Ageing and myeloid-derived suppressor cells: possible involvement in immunosenescence and age-related disease. Age 36:1-12.

Carroll LN, Au AP, Detwiler LT, Fu T-c, Painter IS, Abernethy NF, 2014. Visualization and analytics tools for infectious disease epidemiology: a systematic review. J Biomed Inf 51:287-98.

Chen Y-Y, Huang X-B, Xiao Y, Jiang Y, Shan X-w, Zhang J, Cai SX, Liu J-B, 2015. Spatial analysis of schistosomiasis in Hubei Province, China: a GIS-Based analysis of schistosomiasis from 2009 to 2013. PLoS One 10:e118362.

Duncan DT, Castro MC, Gortmaker SL, Aldstadt J, Melly SJ, Bennett GG, 2012. Racial differences in the built environment - body mass index relationship? A geospatial analysis of adolescents in urban neighborhoods. Int J Health Geogr 11:1-21.

Ferguson RL, Korfmacher K, 1997. Remote sensing and GIS analysis of seagrass meadows in North Carolina, USA. Aquatic Botany 58:241-58.

Fjell A, Walhovd K, 2010. Structural brain changes in aging: courses, causes and cognitive consequences. Rev Neurosci 21:187-221.

Fotheringham AS, Brunsdon C, 1999. Local forms of spatial analysis. Geogr Anal 31:340-58.

Fradelos EC, Papathanasiou IV, Mitsi D, Tsaras K, Kleisiaris CF, Kourkouta L, 2014. Health based geographic information systems (GIS) and their applications. Acta Inform Med 22:402-5.

Getis A, Aldstadt J, 2004. Constructing the spatial weights matrix using a local statistic. Geogr Anal 36:90-104.

Goli A, Kermany FS, Askarian M, 2014. Spatial prevalence of intellectual disability and related socio-demographic factors in Iran, using GWR: case study (2006). Int J Prev Med 5:313-25.

Gonzalez JA, 2013. Geographic Information Systems and Geomatics. In: Pelton JN, Madry S, and Camacho-Lara S, eds. Handbook of satellite applications. New York, NY: Springer New York. pp. 935-54.

Kovacic JC, Moreno P, Hachinski V, Nabel EG, and Fuster V, 2011. Cellular senescence, vascular disease, and aging: part 1 of a 2-part review. Circulation 123:1650-60.

Lopes RAM, Neves KB, Carneiro FS, Tostes R, 2012. Testosterone and vascular function in aging. Front Physiol 3:1-9.

Loughnan ME, Nicholls N, Tapper NJ, 2008. Demographic, seasonal, and spatial differences in acute myocardial infarction admissions to hospital in Melbourne Australia. Int J Health Geogr 7:1-15.

Mena C, Fuentes E, Ormazabal Y, Palomo-Velez G, Palomo I, 2015. Role of access to parks and markets with anthropometric measurements, biological markers, and a healthy lifestyle. Int J Environ Health Res 25:373-83.

Mena C, Fuentes E, Ormazabal Y, Palomo-Vélez G, Palomo I, 2016. Effect of straight-line and road network distances to parks and markets on anthropometric measurements, biochemical markers, and a healthy lifestyle in adult people. Sport Sci Health 12:55-61.
Ord JK, Getis A, 1995. Local spatial autocorrelation statistics: distributional issues and an application. Geogr Anal 27:286-306.

Palomo GI, Icaza NG, Mujica EV, Nunez FL, Leiva ME, Vasquez RM, Alarcon LM, Moyano D, 2007. Prevalence of cardiovascular risk factors in adult from Talca, Chile. Rev Med Chil 135:904-12.

Raskob GE, Angchaisuksiri P, Blanco AN, Buller H, Gallus A, Hunt BJ, Hylek EM, Kakkar A, Konstantinides SV, McCumber M, 2014. Thrombosis: a major contributor to global disease burden. Thromb Res 134:931-8.

Roger VL, Go AS, Lloyd-Jones DM, Benjamin EJ, Berry JD, Borden WB, Bravata DM, Dai S, Ford ES, Fox CS, 2012. Heart disease and stroke statistics - 2012 update: a report from the American Heart Association. Circulation 125:e2-220.

Sarink D, Nedkoff L, Briffa T, Shaw JE, Magliano DJ, Stevenson C, Mannan H, Knuiman M, Peeters A, 2014. Projected ageand sex-specific prevalence of cardiovascular diseases in Western Australian adults from 2005-2045. Eur J Prev Cardiol 23:23-32.

Shaw NT, 2012. Geographical information systems and health: current state and future directions. Healthc Inform Res 18:8896.

Sugumaran R, Larson SR, DeGroote JP, 2009. Spatio-temporal cluster analysis of county-based human West Nile virus incidence in the continental United States. Int J Health Geogr 8:119.

Tanser FC, 2002. The application of GIS technology to equitably distribute fieldworker workload in a large, rural South African health survey. Trop Med Int Health 7:80-90.

Toan DTT, Hu W, Thai PQ, Hoat LN, Wright P, Martens P, 2013. Hot spot detection and spatio-temporal dispersion of dengue fever in Hanoi, Vietnam. Glob Health Action 24:18632-9.

Tsai P-J, Lin M-L, Chu C-M, Perng C-H, 2009. Spatial autocorrelation analysis of health care hotspots in Taiwan in 2006. BMC Public Health 9:1-13.

Varga C, Pearl DL, McEwen SA, Sargeant JM, Pollari F, Guerin MT, 2013. Evaluating area-level spatial clustering of Salmonella Enteritidis infections and their socioeconomic determinants in the greater Toronto area, Ontario, Canada (2007-2009): a retrospective population-based ecological study. BMC Public Health 13:1-17.

Vermeulen A, Goemaere, S, Kaufman J, 1998. Testosterone, body composition and aging. J Endocrinol Invest 22:110-6.

Wieczorek WF, Delmerico AM, 2009. Geographic information systems. Comput Stat 1:167-86.

Zhao F, Cheng S, He G, Huang F, Zhang H, Xu B, Murimwa TC, Cheng J, Hu D, Wang L, 2013. Space-time clustering characteristics of tuberculosis in China, 2005-2011. PLoS One 8:e83605. 